\title{
Atrioesophageal fistula from percutaneous ablation for atrial fibrillation
}

\author{
Lloyd M. Felmly, MD, and Barry C. Gibney, DO, Charleston, SC
}

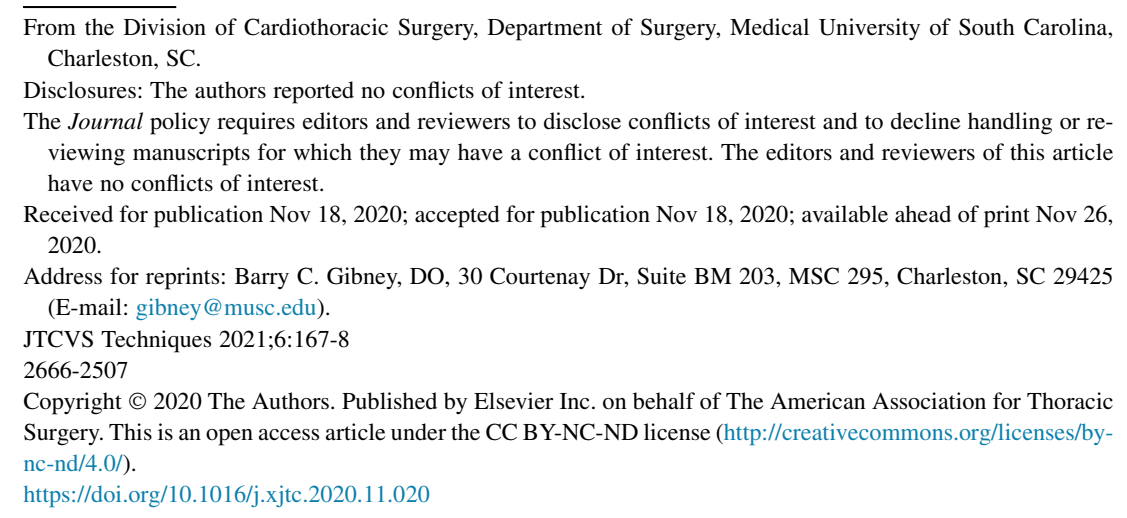

- Video clip is available online.

A 59-year-old male patient with a history of atrial fibrillation who had undergone an atrial ablation procedure 1 month before presented with fever, altered mental status, and right hemiparesis concerning for stroke. Blood cultures grew alpha-hemolytic streptococcus. A computed tomography (CT) scan of the head was negative for embolic or hemorrhagic stroke; however, CT of the chest revealed air in the left atrium (Figure 1, A, arrowhead) and a fistulous connection between the left atrium and esophagus (Figure 1, B, arrow) suggestive of atrioesophageal fistula (AEF). He was taken emergently to the operating room for repair. The imaging suggested the fistula location at the base of the right inferior pulmonary vein, leading to the decision to approach via right thoracotomy. Before positioning, 5 French sheaths were placed in the right femoral vein and artery in the event cardiopulmonary bypass was required. After standard posterolateral thoracotomy, a fifth interspace intercostal muscle was harvested for interposition. As suggested by the imaging, the fistula was encountered at the right inferior pulmonary vein. Before the fistula was sharply divided, the patient was placed head down, and pressure with a sponge stick was used to prevent further hemorrhage and air entrainment. With a pledgeted prolene suture (Ethicon, Somerville, NJ), a single vertical mattress stitch was used to close the fistula. The esophagus was then debrided to healthy tissue, and the muscularis opened to define the

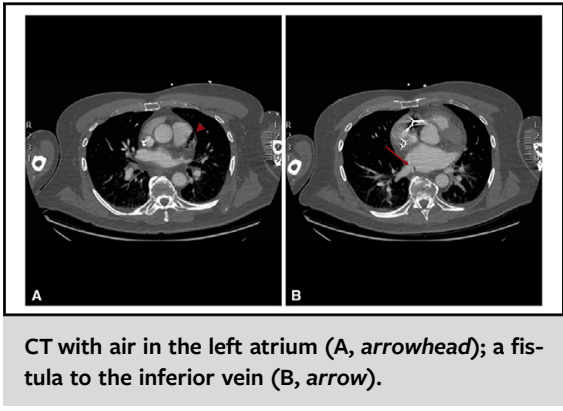

\author{
CENTRAL MESSAGE \\ Atrioesophageal fistulas are rare \\ complications from ablation for \\ atrial fibrillation and present with \\ neurologic symptoms rather \\ than hematemesis.
}

See Commentaries on pages 169 and 170. extent of mucosal injury. A 2-layer closure using vicryl (Ethicon) on the mucosa and silk on the muscle was completed, and the intercostal muscle secured between the esophagus and atrium. Distal enteral access was established at the completion of the operation. The patient's postoperative course was uncomplicated, and he demonstrated gradual neurologic recovery. He was discharged to a neurorehabilitation facility on long-term intravenous antibiotics. At the time of follow-up, the patient demonstrated no neurologic sequelae and was tolerating a regular diet. The patient has consented to publication of his case and images.

$\mathrm{AEF}$ is a rare complication of ablation for atrial fibrillation, with an incidence of $0.1 \%$ to $0.25 \%,{ }^{1}$ but carries mortality reported as high as $80 \% .^{2}$ AEF typically develops within 2 months from the procedure, and presentation is varied-whereas hematemesis or hemoptysis may be the presenting symptom, the most common presenting signs are strokes, seizures, and/or meningitis secondary to bacteremia and air embolism. ${ }^{3}$ Diagnosis can be challenging. Transthoracic echocardiography is usually nondiagnostic, and transesophageal echocardiography and endoscopy should be avoided, as they may aggravate the injury and/or induce air embolism. ${ }^{4} \mathrm{CT}$ is noninvasive and may reveal the fistulous tract or intra-atrial air. Sternotomy, thoracotomy, and ligation of the cervical esophagus are approaches 


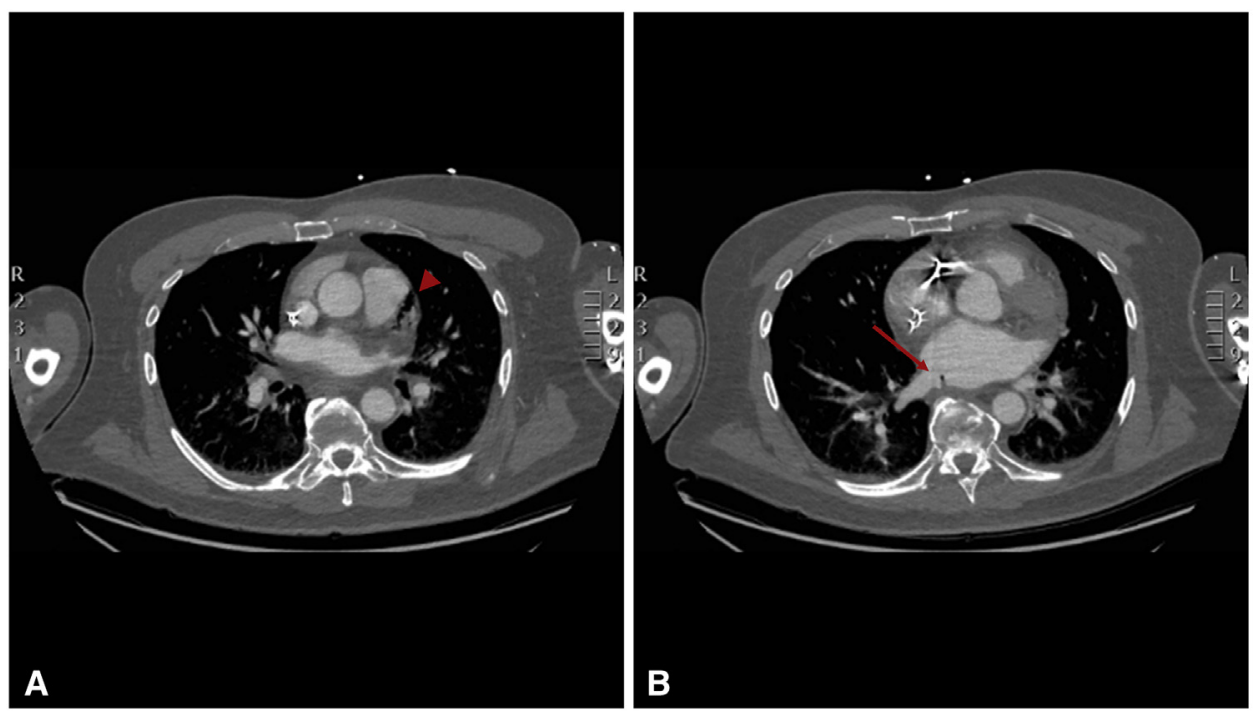

FIGURE 1. Computed tomography with air in the left atrium (A, arrowhead); a fistula to the inferior vein (B, arrow).

that have been reported to facilitate either extracardiac, intracardiac, or spontaneous repair (in the case of esophageal ligation). ${ }^{4}$ Median sternotomy allows arresting and deairing the heart, reducing the risk of air embolus, however, it requires cardiopulmonary bypass. In a patient with acute

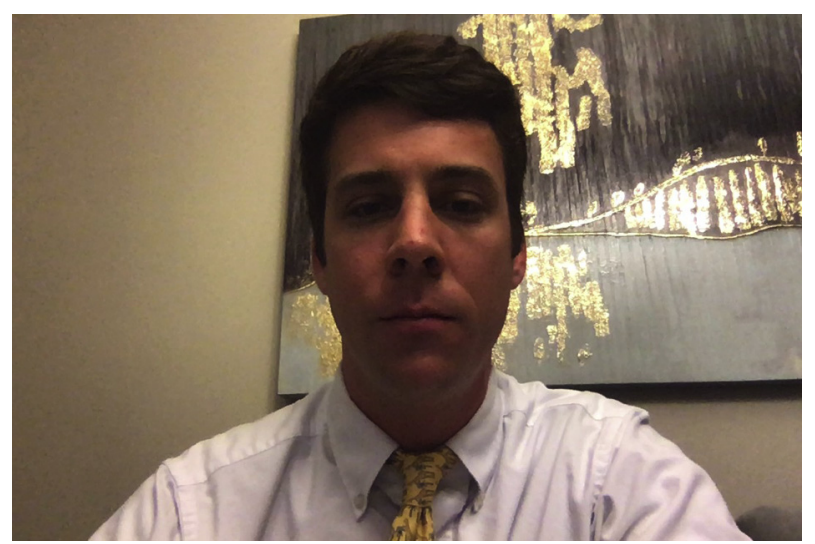

VIDEO 1. Dr Felmly explains the importance of having high index of suspicion and making an appropriate diagnosis in atrioesophageal fistula, as well as discussing management. Video available at: https://www.jtevs. org/article/S2666-2507(20)30709-4/fulltext. neurologic symptoms from acute embolic stroke, this risks catastrophic hemorrhagic conversion. This approach may be justified in situations with no intracranial findings on CT; however, consultation with neurology is advisable. Transthoracic approaches to AEF have classically been described from a left thoracotomy ${ }^{5}$; however, in this case, CT imaging revealed the fistula adjacent to the right inferior pulmonary vein, facilitating repair by influencing us to change our approach (Video 1).

\section{References}

1. Kapur S, Barbhaiya C, Deneke T, Michaud GF. Esophageal injury and atrioesophageal fistula caused by ablation for atrial fibrillation. Circulation. 2017;136: 1247-55.

2. Al-Alao B, Pickens A, Lattouf O. Atrio-oesophageal fistula: dismal outcome of a rare complication with no common solution. Interact Cardiovasc Thorac Surg. 2016;23:949-56.

3. Stöllberger C, Pulgram T, Finsterer J. Neurological consequences of atrioesophageal fistula after radiofrequency ablation in atrial fibrillation. Arch Neurol. 2009; 66:884-7.

4. Julien J St, Putnam JB, Nesbitt JC, Lambright ES, Petracek MR, Grogan EL. Successful treatment of atrioesophageal fistula via cervical esophageal ligation and decompression. Ann Thorac Surg. 2011;91:e85-6.

5. Haggerty KA, George TJ, Arnaoutakis GJ, Barreiro CJ, Shah AS, Sussman MS. Successful repair of an atrioesophageal fistula after catheter ablation for atrial fibrillation. Ann Thorac Surg. 2012;93:313-5. 